\title{
“NEO COLONIALIDAD” Y GESTIÓN DEL RIESGO DE DESASTRES EN AMÉRICA LATINA
}

Jesús Manuel Macías M. ${ }^{\text {* }}$

\section{RESUMEN}

El examen del desarrollo histórico de las organizaciones que los gobiernos en el continente Americano han desplegado para enfrentar desastres, muestra diferencias notables entre Estados Unidos (y Canadá) y el resto de los países latinoamericanos donde éste mantiene evidente rol hegemónico directo (en la relación bilateral y multilateral), e indirecto (a través de las agencias de la ONU incluidas las financieras como el Banco Mundial) en nuestro subcontinente. Esas diferencias pueden ser observadas tanto en la configuración de las administraciones públicas, en término de modelo de organización (Defensa Civil/Protección Civil, Manejo de Emergencias), como en las bases conceptuales del proceso de desastre con fines de intervención (fases del desastre) y la reinvención artificial de los conceptos riesgo, y resiliencia. Esas diferencias suponen una relación de colonialidad ampliamente reforzadas por las organizaciones internacionales mencionadas, a través de acuerdos diplomáticos adoptados en las convenciones internacionales desde Kioto hasta Sendai, etc.. Mientras tanto, los desastres mantienen una tendencia de aumento. Nuestro subcontinente, en su conjunto, asume aún la dominación neocolonial que se expresa precisa y nítidamente en esta esfera del desastre, particularmente en la medida en que la producción de conocimiento mantiene formas subordinadas.

\section{PALABRAS CLAVES}

Desastre, Riesgo, América Latina, Organizaciones internacionales

\section{"NEOCOLONIALISM" AND DISASTER RISK MANAGEMENT IN LATIN AMERICA}

\begin{abstract}
Historical examination about government organizations to face disasters in the American continent shows huge differences between the United States of America (USA), Canada, and the Latin American countries. USA maintains an evident and direct hegemonic role (in the bilateral and multilateral relationship), and indirectly, through UN agencies including financial ones such as the World Bank. These differences can be observed both in the public administrations configuration, in terms of organizational model (Civil Defense/Civil Protection, Emergency Management), and in the conceptual bases of disaster process in order to do interventions (disaster phases). The artificial reinvention of concepts like risk, and resilience also shows that kind of domination. These differences imply a relationship of coloniality largely reinforced by the international organizations mentioned, through diplomatic agreements adopted in the international conventions from Kyoto to Sendai, etc. Latin America, as a whole, still assumes the neocolonial domination that is expressed precisely and clearly in disasters issues, particularly in knowledge production, which maintains subordinate forms. Meanwhile, disasters maintain an increasing trend.
\end{abstract}

1. Centro de Investigaciones y Estudios Superiores en Antropología Social CIESASMéxico, Ciudad de México, México.

*Autor de correspondencia: macserr@att.net.mx

DOI:

https://doi.org/10.55467/ reder.v6i1.81

\section{RECIBIDO}

2 de febrero de 2021

\section{ACEPTADO}

24 de noviembre de 2021

\section{PUBLICADO}

1 de enero de 2022

\section{Formato cita}

Recomendada (APA): Macías M., J.M. (2022). "Neo Colonialidad" y gestión del riesgo de desastres en América Latina. Revista de Estudios Latinoamericanos sobre Reducción del Riesgo de Desastres REDER, 6(1), 9-24. https://doi. org/10.55467/reder.v6i1.81

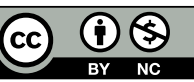

Todos los artículos publicados en REDER siguen una política de Acceso Abierto y se respaldan en una Licencia CreativeCommons Atribución-NoComercial 4.0 Internacional.

Revista de Estudios Latinoamericanos sobre Reducción del Riesgo de Desastres (REDER)

Diseño: Lupe Bezzina

\section{KEYWORDS}

Disaster, Risk, Latin America, International organizations 


\section{INTRODUCCIÓN}

En América Latina la ocurrencia de desastres y las formas en las que se han tratado sus problemas y sus consecuencias, nos hace mirar hacia las características similares que mantienen los países que la componen, los rasgos comunes en la historia y la creación de diferencias... Nada de los antecedentes escapa de las formas actuales en las que se desarrollan conocimientos y acciones frente a desastres y la noción de colonialidad (Quijano, 1992), es quizás la más exacta, como veremos.

Importa acercarse a la comprensión de las respuestas acerca de cómo se han logrado avances, estancamientos o retrocesos, como resultado de las acciones que los países latinoamericanos han realizado para evadir que sus procesos sociales deriven como desastres, y el papel que han tenido los agentes externos (gubernamentales, no gubernamentales y organizaciones internacionales) en todo ello. Los resultados hablan y hablarán por sí mismos como una suerte de evaluación inexorable de todas esas relaciones.

Existen diversos temas alrededor del concepto "América Latina", lo que concierne a este trabajo alude al desastre o más bien al riesgo-desastre. Pero tenemos el interés de mostrar contradicciones entre la realidad y los propósitos manifiestos de interacción en diversos niveles, desde la consideración de países o estados-nación, hasta las organizaciones internacionales. Hay un conjunto de argumentos que se construyen desde la historia, que pasan por ciertas determinaciones del devenir común del subcontinente, en eso que se conoce como dominación, colonial primero y neocolonial después, pero dominación al fin y al cabo. Por todo ello, es el concepto de colonialidad (Quijano, 2014) y neocolonialidad el que tomamos como eje comprehensivo. Dicho concepto está emplazado en la estructura ideológica, es decir, la estructura de la ideología que domina en las sociedades, que sirve para reproducir o continuar las relaciones de dominación asumiendo el doble significado de los desastres: pérdidas y ocasión de flujos de recursos.

Nuestro subcontinente está vinculado entre sí y pasando por Estados Unidos, como el más estricto hegemón (Borda, 2013). Como señala Nourigholamizadeh (2020), se puede identificar un proceso de generación de relaciones hegemónicas de ese país en América Latina, desde la Doctrina Monroe en 1823, hasta la Segunda Guerra Mundial que se caracterizó porque ese país "tomó gradualmente el lugar de las potencias colonialistas europeas en América Latina", prolongando el proceso hasta nuestros días, pero con especial acendramiento, durante la llamada "Guerra Fría".

En ese proceso de creación e instalación de hegemonía, Estados Unidos empleó y generó compromisos económicos y políticos en los países de la región. Precisamente durante la Guerra Fría, fue la disposición de las organizaciones de protección civil/defensa civil, como una forma replicadora de crear cuerpos paramilitares de defensa de eventuales ataques enemigos y para atender problemas de desastres (Victoriano, 2010). También las dictaduras latinoamericanas son un ejemplo de la expansión de los intereses estadounidenses y las fórmulas paramilitares 0 francamente militares de las defensas civiles, lo reflejan.

"...la firma del Tratado Interamericano de Asistencia Recíproca (TIAR), en 1947. Dicho tratado implicaba que las instituciones militares latinoamericanas actuarían como un bloque bélico dirigido. Cinco años antes había creado la Junta Interamericana de Defensa (JID), que tenía como objetivo fomentar entre las repúblicas americanas el intercambio de puntos de vista, el estudio de problemas, la formulación de recomendaciones para la defensa del hemisferio y la colaboración de sus Fuerzas Armadas." (Garzón, 2016, p.28).

Según EOM (2019), las dictaduras de Guerra Fría, "dictaduras de seguridad nacional" en definición de Garzón (2016), más tempranas ocurrieron en Centro América y el Caribe con Anastasio Somoza y familiares, en Nicaragua (1945-1979); Rafael Trujillo y una junta militar en Dominicana (1945-1966); Cuba, con Fulgencio Batista (1945-1959) y El Salvador con Casteneda Castro y una junta militar (1945-1960). Guatemala mantuvo varios dictadores con Castillo Armas, hasta Oscar Mejía (1955-1984); Honduras con Osvaldo López y una junta militar (1963-1981); Haiti con Francois y Jean-Claude Duvalier (1964-1985). En América del Sur se impusieron también dictaduras en Paraguay, con Alfredo Stroessner (1954-1989), en Colombia con Rojas Pinilla (19531957); Venezuela tuvo tres dictadores con Carlos Delgado, Suárez Flamerich y Pérez Jiménez (1948-1958); Ecuador también tuvo tres episodios de dictadura con una junta militar (1963-1966), 
Rodríguez Lara (1972-1976) y otra junta militar (1976-1979); en Argentina varios episodios dictatoriales con Eduardo Leonard y Eugenio Aramburu (1954-1958), luego con J. C. Onganía, Roberto M. Levingston y Lanusse (1966-1972), hasta la junta militar comandada por Rafael Videla (1976-1983). En Brasil la junta militar de la Quinta República (1964-1985); en Bolivia también se registraron varios periodos dictatoriales desde Barrientos hasta Banzer (1964-1977), con una sucesión de 8 presidentes "entre intentos democráticos y golpes de estado" (1978-1982); Perú también mantuvo regímenes dictatoriales con Velasco Alvarado (1968-1975) y Morales Bermúdez (1975-1980); en Chile se impuso dictadura con golpe militar con Pinochet (1973-1990); Uruguay también soportó tres dictadores, Bardaberry, Aparicio Méndez y Gregorio Álvarez (1973-1985). En México no hubo una "dictadura de seguridad nacional", pero si se creó una Defensa Civil bajo mando militar del General Lázaro Cárdenas, de 1942 a 1945, con la finalidad estricta de defender a la población mexicana de eventuales ataques de la alianza del Eje. Fue una Defensa Civil con dirección militar pero integrada por sindicatos y organizaciones civiles con disponibilidad de armamento. (Sánchez-Mejorada, 2001).

América Latina, pasó entonces del dominio colonial al dominio que se conoce como neocolonial bajo la hegemonía de los Estados Unidos (Macías, 2015; Parenti, 2011; Menéndez, 2018). Nourigholamizadeh (2020), sugiere que entre los pilares que preservan y promueven la hegemonía norteamericana son las organizaciones interamericanas y las capacidades militares y económicas. A ello debemos agregar a las organizaciones internacionales en general, dado que la hegemonía de ese país no solo se ha materializado en América Latina sino que ha alcanzado a varios continentes, sobre todo después del colapso de la Unión Soviética en 1991. Los diferentes países latinoamericanos pertenecen o están relacionados doblemente con esas organizaciones, las de carácter interamericano y las mundiales.

En el desarrollo histórico que acompaña a la hegemonía estadounidense, se pueden identificar también otras variantes como la que corresponde plenamente a ese conjunto de políticas y acciones en favor, principalmente, del capital financiero que se denomina neoliberalismo, y que imprimió su marca igualmente en las características del dominio neocolonial. Por ejemplo, la invención del "poder blando" (Jassir, 2015; Romano, 2017; McClory, 2017), "golpe blando" (Ríos, 2018), utilizando los medios de diseminación masiva de datos e informaciones para inducir malestar social y adoptar acciones de cambio de gobernantes, hizo innecesarias a las dictaduras militares para mantener controles en los países latinoamericanos, que son los que nos ocupan. En lugar de gobiernos militares, las tradicionales oligarquías podían funcionar bien como los más altos funcionarios públicos de los gobiernos llegados ahí por elecciones y no por balas. Cambiar para que todo siguiera igual, pero sin violencia tradicional, sería la divisa de Eugene Sharp (2011), uno de los ideólogos del "golpe blando". Ese cambio tendría la legitimidad de la no violencia que había hecho caer en el más absoluto desprestigio al poder militar y contrario a los sentidos humanitarios tan ligados al tema de los desastres.

Por otra parte, las significaciones jurídicas de la soberanía de las naciones también se mostraban como un obstáculo para eficientizar tanto los controles como la extracción de recursos de los países subordinados. Sobre este tema, el de los estados nacionales (EstadoNación), volveremos más adelante, ya que muchas de las políticas y de los "paradigmas" que han surgido desde los años ochenta del siglo pasado, en el campo de estudios de desastres, han marcado el tono de las relaciones con las organizaciones internacionales, siendo un instrumento correspondiente la neocolonialidad actual, precisamente en lo concerniente a desastres.

Organizaciones internacionales como la ONU, son como una suerte de burocracia gubernativa del mundo (Gram-Skjoldager, 2019). Eso también define ciertos términos de las relaciones entre países y entre esas organizaciones y en la esfera del desastre es importante porque éstos no solo significan condiciones críticas, de pérdidas de bienes y de personas, sino también áreas de flujos de recursos de todo tipo, como señalamos al final del capítulo. La relación entre las organizaciones financieras como el Banco Mundial y las diferentes agencias de la ONU, son muy estrechas y alineadas, como veremos.

En este punto conviene revisar los orígenes del sistema financiero mundial. Rajamoorthy (2004, p.1), señala que "En julio de 1944, 44 países se reunieron en la ciudad estadounidense de Bretton Woods, para establecer el sistema monetario internacional de posguerra" y apunta certeramente que "aunque aparentemente era una conferencia de las Naciones Unidas, estuvo 
estrictamente controlada y dirigida por Estados Unidos. Esta reunión histórica que dio origen al Fondo Monetario Internacional y al Banco Mundial marcó el comienzo del dominio de Estados Unidos...".

El Banco Mundial se ha convertido en un grupo de "cinco instituciones de desarrollo estrechamente relacionadas. Su misión evolucionó desde el Banco Internacional de Reconstrucción y Fomento (BIRF) como facilitador de la reconstrucción y el desarrollo de posguerra al mandato actual de aliviar la pobreza en el mundo, coordinándose muy de cerca con su afiliado, la Asociación Internacional de Fomento, y otros miembros del Grupo del Banco Mundial: la Corporación Financiera International (IFC, por sus siglas en inglés), el Organismo Multilateral de Garantía de Inversiones (MIGA, por sus siglas en inglés) y el Centro Internacional de Arreglo de Diferencias Relativas a Inversiones (CIADI)." (BM, 2020).

Hemos señalado recientemente (Macías, 2020a), que la enorme influencia en la economía global del Banco Mundial y las organizaciones financieras que se encuentran en su respaldo, como las aseguradoras Swiss Re, Axa, entre otras 15, así como el papel que desempeña Estados Unidos por medio de la Reserva Federal-Banco Mundial, se encuentra plenamente documentada en Vitali et al. (2011, p.1), en cuanto a la red del control corporativo global: "...las corporaciones transnacionales forman una estructura gigante de "corbata de moño" y que una gran parte del control fluye hacia un pequeño núcleo muy unido de instituciones financieras (privadas). Este núcleo puede verse como una "superentidad" económica que plantea nuevas cuestiones importantes tanto para los investigadores como para los responsables políticos. Newman (2013) basado tanto en el estudio mencionado como en delaciones de una exempleada del Banco Mundial, apuntó de manera particular las relaciones entre el Banco Mundial y la Reserva Federal de Estados Unidos, en cuanto a prácticas de corrupción y control de información e influencias. Por otro lado, la muy estrecha relación entre al Banco Mundial y las organizaciones de la ONU se sintetizan en la Ficha Técnica que presenta el Fondo Monetario Internacional (IMF, 2016):

"El Fondo Monetario Internacional (FMI) y el Banco Mundial son instituciones que forman parte del sistema de las Naciones Unidas y comparten un idéntico objetivo, a saber, mejorar el nivel de vida de los países miembros. Las formas en que encaran la consecución de este objetivo se complementan entre sí: el FMl se ocupa de cuestiones macroeconómicas, mientras que el Banco Mundial se concentra en el desarrollo económico a largo plazo y en la reducción de la pobreza."

Tenemos dibujado un circuito de poder centrado en capital financiero y las organizaciones (gubernamentales y empresariales) que lo controlan.

La relevancia de lo anterior, como señalamos, es mostrar la imbricación de intereses en diversas organizaciones mundiales y la fuerza de la que se puede disponer para impulsar las políticas adecuadas a esos intereses, a propósito de los desastres en América Latina. Los análisis de Toussaint (2007 y 2020), no hacen sino afirmar lo anterior como el recurso de la hegemonía para dominar de manera suave y a veces no tanto, a los gobiernos nacionales y sus políticas. Sobre este aspecto extraeremos algunas lecciones importantes respecto de las implicaciones de las actividades de las organizaciones internacionales en los desastres de América Latina. Como observamos, nuestro subcontinente, en su conjunto, asume aún la dominación neocolonial que se expresa precisa y nítidamente en esta esfera del desastre, particularmente en la medida en que la producción de conocimiento mantiene formas subordinadas, como veremos más adelante, y las acciones que se adoptan, también.

Es necesario aclarar que los vestigios de la dominación colonial y neocolonial a través de la neocolonialidad, deben entenderse más allá de la definición homogeneizadora del concepto país. Cada nación latinoamericana conforma sociedades complejas y contradictorias donde, en general, existen clases sociales y aunque todas las clases asumen la "colonialidad cultural" descrita por Quijano (2014), las clases dominantes son las que sostienen, en esa misma cultura de subordinación, a los factores de reafirmación de sus dominios. La coincidencia de intereses de la clase dominante (económicos y políticos) trasciende la noción de país, y es al mismo tiempo, factor de afirmación de la colonialidad.

Se hace necesario, de nuevo, establecer otra aclaración a propósito del uso del concepto de clases sociales, ya prácticamente inducido al desuso intencional. Apelo a lo señalado por 
Kagarlitsky (2015), en el sentido de que en el transcurso de los tiempos las clases sociales han tenido cambios en algunos aspectos relacionales, según las definiciones del marxismo tradicional, pero siguen existiendo y su naturaleza lo confirma en toda forma de ubicación de las diferencialidades sociales. Las "teorías del fin de las clases sociales", como concluye Amorim (2015, p.34), "desarrolladas sobre todo en Europa Occidental y Estados Unidos" fueron importadas al mejor estilo de neocoloniedad por intelectuales brasileños y en general de América Latina, para analizar movimientos y conflictos sociales, con lo que "se explicitó no un debate teórico-crítico sino, en la práctica, una estrategia política e ideológica que colocó a toda una tradición analítica heterogénea en un nivel límite de abstracción."

La esfera de los desastres tiene varios mantos: el humanitario, el de intervenciones financieras, de flujos de mercancías, de flujos de conocimientos y es, también, un escaparate de relaciones que reproducen la colonialidad señalada por Quijano (2014).

Los intercambios de conocimiento ilustran muy bien esa colonialidad, advertida por Quijano, con la imposición de los propios "patrones de producción de conocimientos y significaciones" de las naciones dominantes. Lo que se ha visto respecto de las organizaciones internacionales y el tema de desastres, nos ilumina acerca de los vehículos que han estado presentes en nuestra América Latina. Las propuestas conceptuales y de acción respecto de la gestión de riesgo de desastre, el papel de gobiernos y organizaciones no gubernamentales, las escalas del desastre, los arreglos internacionales, los conceptos de vulnerabilidad social, resiliencia, y desde luego, lo concerniente a las bases conceptuales justificadoras de la utilización de recursos financieros para el "desarrollo". Todo esto frente a los resultados de esfuerzos o simulaciones conjuntas, en la reducción o no, de los desastres.

Los propósitos del hegemón de América Latina, como vimos son directos a través de las relaciones intergubernamentales y su agencia de Ayuda (USAID) e indirectos con todo el sistema de la ONU, incluido el Banco Mundial y sus organismos financieros asociados, que implican a un ejército de especialistas en riesgo de desastre, con vínculos contractuales orgánicos o eventuales. No sobra mencionar que el conjunto de instrumentos de la hegemonía incluye a organizaciones académicas y editoriales reguladoras del mercado de la transmisión de conocimiento. Para cada uno de esos instrumentos y relaciones, las sociedades latinoamericanas tienen sus correspondientes, mediados precisamente por la razón de la colonialidad.

Existe una cantidad apreciable de literatura dedicada a los temas del área de riesgo desastre, de la que usamos algunas referencias que se consignan aquí, y que ampliaremos más adelante. En este punto conviene advertir algunos de los "pilares" que han sostenido buena parte de la relación de América Latina con países hermanos y con las diversas organizaciones internacionales y otros estados.

Los pilares mencionados han sido establecidos básicamente por la hegemonía y sus recursos instrumentales de los que se ha hablado y son:

1. Desestimación diferenciada de gobiernos nacionales en favor de Organizaciones No Gubernamentales para, entre otras cosas, impulsar demandas públicas de medidas de gestión de riesgos (CGCED, 2002; Albala-Bertrand, 1993 y 2014; Freeman et al., 2002, Henderson, 2004);

2. Difusión de beneficios potenciales de gestión de riesgo creando "resiliencias";

3. Reforzar la idea de vinculación de los desastres con el desarrollo, donde la consideración del no desarrollo es causa de los desastres que debe corregirse porque genera pobreza (Hallegatte et al., 2017). La noción de "desarrollo" es referida solo al crecimiento económico (Zabalo, 2002); y,

4. Fomento de políticas y acciones de Gestión de Riesgo (gestión integral del riesgo de desastre) con gobiernos y a nivel local con organizaciones no gubernamentales.

\section{SOBRE CUESTIONES DE MÉTODO}

Siguiendo la noción de metodología de Dussel (1966, p.19) respecto a que "los presupuestos metódicos son condicionantes de todo el resto", nos proponemos un método, el análisis de una estructura de relaciones que permita "entender" la realidad, comprenderla para acceder a una elucidación del conjunto de factores que son comunes a los países latinoamericanos, el "nosotros 
mismos como grupo cultural histórico" de Dussel. Igualmente tratamos de que dicha estructura metódica contenga la cualidad de englobar, sistematizar y abarcar, lo más posible, los fenómenos más relevantes pertinentes al análisis de las ciencias sociales.

En este esfuerzo metodológico está involucrado una combinación de ejercicios, tanto de corte estrictamente académico (revisión de literatura, discusiones seminales teórico-conceptuales) como de investigación empírica, pero también están presentes elementos de praxis política. La conceptualización general acerca de la colonialidad y lo relativo a las nociones del riego-desastre, han sido el eje que da luz a los intercambios de conocimientos y acciones a través del tiempo que realizan tanto organizaciones sociales tanto de corte académica como gubernamental, de organizaciones internacionales, de organizaciones no gubernamentales.

América Latina es aprehendida en su dimensión abstracta de países con cierta identidad incluyente, es decir que comparten rasgos comunes de un proceso histórico, porque fueron colonias de naciones europeas para luego seguir bajo relaciones de dominación neocolonial. Bajo esa consideración procesual se asume la idea de colonialidad de Quijano para explicar la reproducción del tipo de relaciones que se han mantenido entre sí y con el exterior. La idea de estado nacional es considerada de gran importancia, por diversas razones, pero principalmente por lo que tiene que ver con la noción de soberanía territorial. También se considera, en el conjunto de relaciones, al rol de las clases sociales, sobre todo las dominantes. El nivel de integración de espacios geográficos y sociales tomando de manera relevante la ideología. Sus relaciones con las organizaciones internacionales, son consistentes con la forma ideológica propia de la colonialidad y la variante derivada del orden neoliberal, que ordena las instancias internacionales bajo un dominio de los Estados Unidos. Se considera también el rol de las actuaciones "intelectuales" para afianzar lo que se llamó "Globalización del mundo" cuyo rasgo común fue reducir las soberanías y crear la "declinación" de los Estados Nacionales, y junto con ello va el impulso a lo "local" como una muestra de que los gobiernos nacionales no son confiables. Este tema es relevante por cuanto conduce a otra discusión que apenas tocamos lateralmente aquí a propósito de un ejercicio de investigación curricular sobre formación de posgrado para la gestión de desastres: las responsabilidades de Estado frente a los desastres.

El planteamiento metodológico, por etapas sería de la siguiente manera: 1) desarrollo del concepto de colonialidad de Quijano y de Dussel para la neocolonialidad, en revisión a los antecedentes de las producciones intelectuales y materiales en América Latina relacionados con riesgo-desastre; 2) análisis de las organizaciones sociales nacionales e internacionales vinculadas al rol de favorecer al hegemón norteamericano, como la concreción de la neocolonialidad; 3) configuración del marco de acción del hegemón y sus instrumentos en América Latina considerando algunas determinaciones históricas como el Neoliberalismo, que necesitó la llamada globalidad del mundo en contra de los Estados Nación-gobiernos-soberanía para favorecer organizaciones de la "sociedad civil"; 4) la formación de recursos humanos en el subcontinente para reducir desastres, que se contrasta con lo desarrollado en Estados Unidos y Canadá como entidades nacionales americanas; 5) Razones o conclusiones.

\section{ADELANTANDO RESULTADOS DEL EJERCICIO DE ANÁLISIS "NEO COLONIALIDAD" Y GESTIÓN DEL RIESGO DE DESASTRES EN AMÉRICA LATINA \\ Organizaciones internacionales-gobiernos nacionales}

La organización del Decenio Internacional para la Reducción de Desastres Naturales (DIRDN) por la ONU, es un punto de partida importante para observar el rol de las organizaciones internacionales en relación con los gobiernos y los desastres. Se ha sugerido que los elementos justificatorios de ese esfuerzo internacional empezaron desde antes de la Guerra Fría, pero que en fue una iniciativa más reciente emanada de científicos (Prior y Roth, 2015). Schemper (2019) consideró que el DIRDN tuvo originalmente un marco científico centrado en las amenazas, en el contexto del socorro en casos de desastre, que lo alejaba de motivos políticos porque su planteamiento de origen era la reducción de las amenazas naturales. Como se modificó su objetivo para enfatizar la reducción de desastres, también sirvió, según el autor, para desvincular el Decenio de su componente humanitario, que fue confiado al sucesor de UNDRO, el Departamento de Asuntos Humanitarios (ahora Oficina de Coordinación de Asuntos Humanitarios, OCHA) en 1991. Lo anterior refleja visiones neutrales acerca del funcionamiento de las organizaciones de la ONU, atribuyendo al DIRDN un despegue científico natural. Lo que se debe tener en cuenta 
es que hubo también reacciones de científicos sociales llamando la atención a la parcialidad del planteamiento inicial y probablemente el cambio de objetivo de reducir solo amenazas naturales para considerar planamente a los desastres (Bates, Dynes y Quarantelli, 1991).

Como señalamos anteriormente, la creación de la Liga de las Naciones-ONU tiene implícita la existencia de una burocracia internacional, es decir, un cuerpo de servidores públicos internacionales que no necesariamente han sido servidores internacionales en abstracto, sino que han tenido que observar intereses o presiones de las elites de las potencias (Gram-Skjoldager, 2019). Revet (2011a) identificó muy bien el papel de las organizaciones internacionales como ámbito de promoción de ideas dominantes o consensuales, entre otras cosas, considerando ese sector como clave en la consolidación de consensos o "paradigmas", adoptados luego hasta por gobiernos nacionales:

"Históricamente este mundo [de los desastres] fue creado desde los actores e instituciones del espacio humanitario internacional, y se centró al principio en las operaciones de socorro. Ahora, crece progresivamente y se diversifica, incluyendo la noción de prevención, lo que trae como consecuencia la apertura a actores inicialmente relacionados con cuestiones de desarrollo o con cuestiones ambientales. Produciendo discursos, herramientas, normas, formas de pensamiento y creencias propias, este espacio internacional de la gestión de riesgos y de desastres naturales ejerce hoy en día una influencia decisiva sobre la formulación de las problemáticas, la estructuración de las políticas nacionales y locales, la financiación de los proyectos de las organizaciones no gubernamentales y las posturas de los diversos actores (Revet, 2011a, p.552)."

En el reservorio argumental de "consensos" de las mencionadas organizaciones internacionales, acerca de porqué los países pobres sufren más en desastres, invariablemente se encuentran las razones siguientes: son más propensos a la ocurrencia de las amenazas, están más mal preparados que los países desarrollados y sus gobiernos tienen burocracias atrasadas y mal financiadas (CGCED, 2002). Ello, por cierto, ha funcionado para que, con el noble fin de la "ayuda" a los pobres y para la recuperación de los impactos desastrosos se registre el fenómeno bien caracterizado por Revet (2011b, p.11): “... el continuo aumento de organizaciones transnacionales o internacionales como las entidades de las Naciones Unidas, las agrupaciones orientadas religiosamente y el Banco Mundial. Con el declive de la importancia del Estado-nación se deben esperar cada vez más actores sociales".

Desestimación diferenciada de gobiernos nacionales en favor de organizaciones no gubernamentales para impulsar demandas públicas de medidas de gestión de riesgos

La afirmación de Revet, líneas arriba, es una excelente observación acerca del argumento o "teoría" del "declive de los estados nacionales" sobre lo que se ha elaborado la justificación de pasarse por alto a los gobiernos para impulsar a organizaciones no gubernamentales, y fue un propósito inicial para preparar la expansión del Neoliberalismo: no a los estados nacionales, si a la globalización.

Desde inicios de los años noventa del siglo pasado, se advertía esa consideración negativa hacia los gobiernos nacionales de los países pobres, esos gobiernos que la hegemonía neocolonial había instalado para su control. Albala-Betrand (1993) en el análisis de la "economía política" de los grandes desastres, advirtió que dejar su política en manos de los sistemas gubernamentales existentes, solo fortalecería "estructuras estrechas de poder" y recomendaba la inserción de actores no gubernamentales. Habría que insistir que esas "estructuras estrechas de poder" son las estructuras que impusieron en la fase neocolonial y que dejaron se ser útiles, lo que hace girar la atención y las iniciativas locales. "Quienes apoyan esta teoría creen en la conveniencia de reducir el riesgo de los peligros de la naturaleza a través de proyectos conducidos en el ámbito comunitario y programas desarrollados por organizaciones no gubernamentales (ONG). Esta forma de abordar la gestión del riesgo puede no ser integral, pero atiende directamente las necesidades identificables y permite actuar a las poblaciones locales." (Zapata, 2011, p.1).

Como mencionamos antes, también las recomendaciones de los organismos financieros son para introducir esquemas de organización considerando gobiernos pero impulsando organizaciones no gubernamentales, lo significa la propuesta de Freeman et al. (2002) para constituir "Sistemas Nacionales para la Gestión Integral del Riesgo de Desastres. Estrategias 
Financieras para la Reconstrucción en Caso de Desastres Naturales", donde describen lo que a su juicio son los componentes de dichos "sistema", subrayando que además de las interconexiones de "instituciones", son los mecanismos financieros, y las "normas y las políticas" que definen la manera en que se debe abordar la "gestión del riesgo de desastres" de un país, aclarando que esa interconexión interinstitucional puede ser formal o informal y advirtiendo que: "comúnmente se cree que para que un sistema nacional para hacer frente a los desastres sea integral, los gobiernos nacionales deben participar activamente en la creación y puesta en práctica de un sistema formal. Sin embargo, no existe en la literatura una única opinión sobre la conveniencia de depender de los gobiernos nacionales como base correcta de un programa integral." (Zapata, 2011).

Difusión de beneficios potenciales de gestión de riesgo creando "resiliencias"

Otro de los fundamentos en las que las intervenciones de los organismos financieros internacionales tienen asidero es una concepción, digamos, neutra de los factores y causas de la pobreza y la dimensión que adopta, en ese sentido, la resultante positiva de esas intervenciones. Básicamente la consideración sobre la pobreza se limita a observar la relación entre las consecuencias de la pobreza y los factores del riesgo-desastre, derivando recomendaciones difícilmente asimilables en términos de su utilidad. Omiten las verdaderas causas de la pobreza que se centran en la explotación del trabajo y la profunda desigualdad social que ocasiona históricamente esa doble relación: la explotación doméstica y la explotación neocolonial. A pesar del cambio de "paradigmas" del "Fin de la historia", de la declinación de los estados-nación, la pobreza se mantiene en un rango de población que va creciendo inexorablemente. Por ello, se ha erigido un concepto que busca justificar la existencia de intervenciones externas "para el desarrollo" y para "reducir desastres": la resiliencia. Como veremos al final de esta contribución, los resultados de todos los esfuerzos internacionales, nacionales, locales, etc., encaminados a la reducción de desastres, no han tenido éxito. ¿Cómo justificar entonces la continuación de políticas "más de lo mismo"? Sin duda la justificación sería hacer más resilientes a los que sufren desastres y a los que están en riesgo de calamidad, algo así como una relación directamente proporcional entre la resiliencia y la pobreza: aumenta la resiliencia y disminuye la pobreza, veamos:

"Otro hallazgo importante es que en muchos países, especialmente los de bajos ingresos, las opciones muy prometedoras para aumentar la resiliencia de las personas son también una buena reducción de la pobreza y políticas de desarrollo, como la inclusión financiera o la protección social. En estos países, es muy fácil alinear las prioridades de desarrollo con la gestión del riesgo de desastres y aprovechar las sinergias entre estos dos objetivos." (Hallegatte et al., 2017, p.175).

La base de los supuestos de los expertos del Banco Mundial sigue siendo una suerte de "circulo vicioso" con la pobreza y los desastres. Los pobres son más propensos a sufrir desastres y éstos mantienen pobre a la gente, no es la explotación del trabajo, no se involucra otro tipo de relación socioeconómica en el conjunto argumental de las intervenciones. La resiliencia es una condición a crear externamente.

\section{Reforzar la idea de vinculación de los desastres con el desarrollo, donde se asume que el no desarrollo es la causa de los desastres, que debe corregirse porque genera pobreza}

Las visiones neocoloniales acerca de la pobreza y sus causas se parecen mucho a la racionalidad de las "damas de la Caridad" (AIC, 2010). Los pobres sufren los desastres porque son pobres y el Banco Mundial, por centrarse en éste, debe cumplir su propósito de reducir la pobreza, en los pobres está la misión: "explorar los instrumentos para la transferencia del riesgo de desastres y su relevancia para proteger a los pobres de los impactos de desastres... el papel que pueden desempeñar los mecanismos de transferencia de riesgos en la promoción de la sensibilización y la inversión en medidas de mitigación de desastres, y su eficacia potencial para aislar a los pobres de los impactos de los desastres. (Wharton, 2000, p.8).

Como afirma también Henderson (2004), el riesgo generalizado de devastación está en las naciones pobres, lo que quiere decir que se deben esperar, como ha sido, la pérdida de muchas vidas de personas y de sus bienes. Todo como resultado de los desastres (humanos y "naturales"). Las causas son el "estrés socioeconómico" el envejecimiento, la infraestructura física inadecuada, mala educación y mala preparación para los desastres, pero también "insuficientes recursos fiscales y económicos para aplicar cuidadosamente los componentes de preparación, respuesta, mitigación y recuperación de la gestión integrada de emergencias" (Henderson, 2004, p.103). 
Fomento de políticas y acciones de Gestión de Riesgo (gestión integral del riesgo de desastre) con gobiernos y a nivel local con organizaciones no gubernamentales

Cavani y Revet (2015), observaron un rasgo muy interesante de las formas latinoamericanas que responden a los esfuerzos internacionales por mejorar la gestión del riesgo de desastres. Analizaron el rol de la organización no gubernamental denominada La Red, surgida en 1992 y ubicaron la ulterior asimilación de varios de sus fundadores en organismos internacionales (EIRD, Banco Mundial, etc.). Las autoras advierten el sentido de los proyectos que se impulsaron desde esa organización, que, de manera particular, se refieren a "la participación de las comunidades en el proceso de prevención y reducción de riesgos", atribuyéndoles un "enfoque anglosajón" relacionado con lo que en inglés se conoce como "Community based disaster risk management" (CBDRM), lo que asocia a dicha organización con la línea del Banco Mundial (GFDRR, 2014; Van Niekerk et al., 2017).

\section{Impulso a la Gestión Integral del Riesgo de Desastres (GIRD)}

Las razones que motivaron la organización del DIRDN y de las conferencias mundiales de la ONU (Kioto, Hyogo, Sendai) tenían un referente en el acuerdo de que los gobiernos nacionales no tenían ni las capacidades ni los recursos para enfrentar amenazas de desastres, fue claro que disponían de arreglos organizativos vinculados a la respuesta de "ataque enemigo" (Dynes, 1994), y que se caracterizó luego como un "modelo reactivo". En esa crítica hubo coincidencias en prácticamente todas las esferas de los actores (científicos, burócratas internacionales, operativos nacionales...) y se impulsó la idea de impulsar una alternativa a ese modelo reactivo-modelo militar, y se habló de las gestión o manejo de desastres, luego "gestión del riesgo" y finalmente "gestión integral del riesgo de desastres".

Es importante señalar que los orígenes de ese modelo alternativo a las defensas civiles/ protecciones civiles, que se asumió para su difusión en América Latina, se encuentran en los Estados Unidos y en todo el proceso de reorganización de la administración pública federal por crear un Sistema Federal de Manejo de Emergencias (SFME) en 1979. En el nuevo sistema norteamericano se adoptó una conducción plenamente civil y directamente alojado en la oficina del Presidente (Casa Blanca). Ese proceso no estuvo exento de conflicto interno con el interés de militares que siempre pugnaron por un énfasis en las amenazas de terrorismo, que luego desembocaría en el desmantelamiento del SFME para crear el Departamento de Seguridad Interior (Home Land Security Department), donde los militares volvieron a asir el control de la organización bajo el contexto de los ataques terroristas ocurridos el 11 de septiembre de 2001 tanto en Nueva York como en Washington (Macías, 2016).

Lo que podemos considerar como el modelo civil (en inglés: integrated emergency management) proveniente de EEUU, se ofreció luego como la alternativa a las defensas civiles en América Latina y fue en el marco del DIRDN que tuvo lugar un esfuerzo impresionante de capacitación en ese modelo civil a través de la ONU (con sus agencias PNUD y UNDRO en 1990), sustentado al Centro de Manejo de Desastres de la Universidad de Wisconsin-Madison. Dicho centro estaba asociado a una empresa consultora de formación y capacitación, denominada INTERTECT que había creado Frederick Cuny, un profesional de asuntos humanitarios que trabajó para el gobierno de Estados Unidos y fue impulsor del enfoque que centraba en la relación entre los desastres y el desarrollo, la vía para reducir sus impactos adversos. La plataforma de capacitación en el manejo integrado de desastres se denominó: Programa de Capacitación en Manejo de Desastres (DMTP, por sus siglas en inglés), y fue formalmente aprobado "por la Resolución de la Asamblea General 46/182 en su 78a Reunión Plenaria celebrada el 19 de diciembre de 1991. El DMTP ha sensibilizado sobre la necesidad de una gestión más eficaz de crisis y desastres para reducir los riesgos y vulnerabilidades" (WHO/OMS, 2004).

El nuevo modelo civil de gestión de desastres fue esencialmente gubernamental y específicamente organizacional, pues mantuvo tres pilares esenciales, acordes con la organización federal de los Estados Unidos: (1) de "gobierno compartido" entre tres órdenes - federal, estatal y municipal y dando una ubicación al sector no gubernamental, denominado "sector privado"; (2) la organización debía intervenir en cuatro fases del manejo de desastres (mitigación, preparativos, respuesta y recuperación), lo que implicaba no solo atender las "emergencias" como se podría interpretar de la denominación del modelo (emergency management), sino injerir también desde las políticas y acciones preventivas donde todas las fases implican la atención y acción del proceso de 
desastre; (3) el modelo definía un orden de consideración de "todas las amenazas", lo que suponía ejercer planificación y operaciones en todas las fases para todas las amenazas y no tratarlas de manera separada. La lógica era muy sencilla y se basaba en que básicamente cualquier impacto desastroso generaba casi las mismas demandas de atención de la población afectada: búsqueda y rescate, atención médica, refugio de emergencia, etc. El modelo funcionaba para cualquier escala de impacto desastroso, aunque tenía previsiones de planificación diferenciadas. Todo lo anterior se construyó para cumplir con una obligación de Estado de proteger la vida y los bienes de los miembros de la sociedad. En este sentido, es clara la naturaleza gubernamental de este modelo.

En el transcurso del DIRDN, se fueron registrando cambios en los esfuerzos relacionados con el enfoque de "capacitación" para el manejo de desastres. El DMTP, perdió impuso y empezaron a surgir otras iniciativas que buscaban cambio de enfoque, por ejemplo, desestimar el uso del concepto "desastre" y, en su lugar, usar el de "riesgo"; disminuir el énfasis en los esfuerzos con gobiernos y ponerlos en los niveles locales (Lavell, 2003), hablando de comunidades o de organizaciones no gubernamentales de acción local.

Hemos interpretado esos giros, como correspondientes con algunas necesidades del sector financiero mundial (Macías, 2020b), por ejemplo, con las exigencias del Banco Mundial para ampliar el mercado de seguros y darle salida a la sobre-capitalización de las aseguradoras y reaseguradoras originado por la exigencia del gobierno norteamericano para resarcir la falencia de ese sector, en el caso de la atención de las consecuencias del impacto del huracán Andrew en el sur de Florida en 1992.

En el propósito de los esfuerzos internacionales para incidir en el cambio del modelo reactivo al preventivo para enfrentar desastres, la propuesta señalada antes de Freeman et al. (2002) para los acceder a mejores "sistemas nacionales para la gestión integral del riesgo de desastre", es muy interesante porque toma elementos del emergency management, como las cuatro fases, para introducir otras dos concentradas en actividades que exigirian consumo de seguros. Se mantuvo el adjetivo de "integral" y sugirió una integración de organizaciones amplia, probablemente trascendente de los organismos gubernamentales.

Los ejemplos mencionados son consistentes con desestimar a los gobiernos, impulsar el consumo de seguros y reaseguros, lo que tuvo éxito en diversos gobiernos nacionales como el de México, donde se adoptó la adquisición de "bonos catastróficos" y se ajustó la legislación de los fondos financieros para atender desastres, obligando a gobiernos municipales y federales a acudir al aseguramiento de infraestructura. No sobra advertir que la "venta" de seguros ha acudido a una fórmula falaz que se ha denominado "transferencia de riesgo" y que se refiere en realidad a una transferencia parcial de pérdidas. Cualquier fórmula conceptual sobre "riesgo" puede demostrar su imposibilidad de ser transferido. Pero la palabra "riesgo" ha ocupado desde entonces un lugar central.

Colonialidad es el concepto clave para entender la aceptación de gobiernos y otras organizaciones sociales (incluidas las académicas) en América Latina, de esas acciones impulsadas y sostenidas por los organismos internacionales.

\section{DISCUTIENDO RESULTADOS PARA APROXIMARSE A CONCLUSIONES PROVISIONALES}

La GIRD que se ha buscado impulsar en América Latina, tiene contradicciones de inicio y que se infieren por lo señalado en el apartado anterior. Sin embargo, es importante documentarlas, a través de un ejercicio comparativo. ¿Cómo se sostiene la GIRD en los países latinoamericanos en términos conceptuales y de acción? Nosotros suponemos que debe ser a través de la formación de recursos humanos que tiendan a una especialización profesional.

La exacerbación de la demanda del Banco Mundial por ampliar el mercado de seguros ha llegado a forzar diversas expresiones sobre la GIRD que no necesariamente conforman un modelo que apunte a una profesionalización necesaria, sino un conjunto de propuestas que desbordan descripciones de eventuales procesos y buenos deseos como el de Narváez et al. (2009), pero no tienen una referente organizacional real. Por ejemplo, la invención de conceptos tales como gestión "reactiva" "preventiva", "correctiva", es probablemente una buena analogía de la ingeniería de mantenimiento, pero no tienen correspondencia con instancias reales de la sociedad. De la misma manera que las propuestas de gestión local del riesgo, pueden tener algún sentido para ciertas sociedades rurales y para determinadas épocas del año, pero se enfrentan con el desconocimiento 
real de cómo funcionan las sociedades y sobre todo los segmentos pobres, que están obligados vitalmente a concentrar sus atenciones en cómo acceder al pan cotidiano, antes que a fórmulas de "reducción de riesgo de desastre".

\section{Comparación de soportes para la GIRD en América}

En 2018, realizamos un ejercicio de revisión de currícula profesional y de posgrado (Maestría) entre América Latina y la de habla anglosajona (Estados Unidos y Canadá), encontramos lo siguiente:

La noción de Gestión Integral del Riesgo de Desastre (GIRD), como se ha reiterado, es actualmente la que tiene posición privilegiada en el ámbito de la prevención y atención de desastres, pero dicha noción representa un problema: en América Latina aparece como un desarrollo reciente completamente desvinculado de sus precedentes conceptuales y organizacionales que se han identificado, como ya se dijo, en los Estados Unidos, en la fórmula de emergency management. Ello representa una cierta desventaja y un efecto de confusión ya que los alcances de su aplicación, sobre todo en los términos de profesionalización, reflejan ciertas desviaciones preocupantes que se proyectan en otras ofertas formativas en la GIRD y en la Reducción de Riesgo de Desastres (RRD), un ejemplo son los análisis de ofertas curriculares en América Latina, como los realizados por Lara et al. (2014) y Lara (2016), para el caso de la GIRD y de la Estrategia Internacional para la Reducción de Desastres (EIRD, 2009) para el caso de la RRD.

Una vez que aclaramos la relación de la GIRD con sus antecedentes en el modelo norteamericano de emergency management, se pueden observar importantes posibilidades comparativas. El problema de la existencia de esos dos modelos conforma una tendencia inercial en las prácticas y propósitos que definen una necesidad de cambio con la adopción de la GIRD, como clave para transitar del modelo reactivo al preventivo.

\section{América Latina}

Para el caso de América Latina, se consultaron los dos estudios mencionados que realizaron una suerte de "inventario" de las ofertas formativas relacionadas en GIRD (Lara et al., 2014 y EIRD, 2009). Ambos documentos son resultado de investigaciones realizadas bajo encargo, por la EIRD y obedecen prácticamente a un esquema que busca las ofertas curriculares relacionadas con la GIRD, en el universo de Instituciones de Educación Superior (IES) en el subcontinente. EI estudio de Lara (2016) ofrece, además de información sobre las ofertas curriculares, una suerte de clasificación, así como un ordenamiento primario de "enfoques" de esas mismas ofertas.

En el análisis comparativo, Lara et al. (2014) destacan que México es el país con mayor número de IES (1892) en toda América Latina y sin embargo tiene el cuarto lugar en ofertas curriculares relacionadas con GIRD. El caso de Colombia destaca por tener el mayor número de ofertas curriculares relacionadas con la GIRD (14) y un número menor de IES (349). Lara encontró una oferta total de 81 programas vigentes relacionados con la GIRD en todo el subcontinente, y en la tipología de la oferta académica encontró que existen 17 maestrías. Una revisión de las ofertas señaladas, indica claramente una orientación dominante hacia las ingenierías y/o las ciencias naturales, cosa que llamó la atención en el análisis realizado en 2009 a solicitud de la EIRD, por las implicaciones advertidas en la cita siguiente:

En el informe final de la conferencia hemisférica hace alusión sobre las varias iniciativas de carácter universitario, entre ellas las que impulsan la creación de maestrías en temas relacionados con el manejo de los desastres y la reducción del riesgo; al igual hace mención de que en las universidades sigue predominando las geociencias, la ingeniería y los aspectos estructurales de la mitigación, pero no el tema de los preparativos y la capacidad de respuesta, se comenta también sobre el poco desarrollo de currículos sobre reducción de riesgos en las facultades de ciencias sociales (EIRD, 2009).

Se puede señalar que hay una diferencia importante no solo en la calidad y cantidad de esas ofertas en países como Estados Unidos y Canadá, con respecto a los países latinoamericanos. Esa diferencia está indicada justamente porque aquellos mantienen una relación con una profesión consolidada, y, en el caso de América Latina, la profesión tiene aún una forma embrionaria, es decir, está en proceso de formación profesional.

Como se observa, se puede identificar una cierta diferenciación entre la GIRD en América Latina, y la propia de los Estados Unidos y Canadá. Ahora bien, la comparación sugiere que las 
diferencias se encuentran en los procesos de inclusión y desarrollo de la propia GIRD, que tiene un cierto reflejo en la mayoría de los contenidos de los programas curriculares. En este sentido se observan dos fenómenos interesantes:

1. En América Latina la GIRD tiene un importante impulso de organizaciones internacionales (EIRD, UNESCO, PNUD) y principalmente financieras (Banco Mundial, Banco Interamericano de Desarrollo) y de ayuda exterior (Cooperación Italiana, Cooperación Alemana, Comisión Europea-PREDECAN, etc.), que han incorporado recursos técnicos y financieros a través de ONGs y, probablemente en menor proporción, con los gobiernos nacionales, provinciales y locales, por tanto ello sugiere un distanciamiento hacía la profesionalización ligada a las funciones públicas.

2. En América Latina se ha identificado un cierto énfasis en el desarrollo de la GIRD de "nivel local" o comunitario, y, como se ha señalado antes, también en EEUU y Canadá se reconoce que la "comunidad" es el primer ámbito de reacción. Sin embargo, es necesario hacer notar que el sentido del término "comunidad", "comunitario" o "nivel local", en América Latina se asocia con la ausencia de relación con gobiernos de ese nivel o superiores. Lo contrario para el caso de EEUU y Canadá. En México, la "comunidad" se asume como un ámbito más reducido que el Municipio y aunque los "órdenes de gobierno" jurídicos solo consideran tres ámbitos, federal, estatal y municipal, a nivel de las comunidades, barrios, núcleos agrarios, ejidos, etc., se reconocen medios de gobierno formalmente.

\section{Estados Unidos y Canadá}

El desarrollo de la profesionalización de esa función tiene un reflejo en la oferta curricular. Marks (2005) desarrolló un profundo análisis de la oferta curricular para clarificar los planos de las competencias respectivas, y encontró que para el nivel de maestría se habían ofrecido 70 programas desde 1998, de los que obtuvo información directa en 32, con el contenido directo de GIRD y otros 15 programas del mismo grado con contenidos relacionados.

Kapucu (2011, p.503) actualiza los datos señalando que "existen 173 programas de gestión de emergencias en universidades: 59 certificados, menores, títulos; 39 grados asociados; 26 licenciaturas; 42 programas de nivel de maestría; y 7 programas de doctorado. Además, 32 programas de manejo de emergencias están bajo diseño o en desarrollo."

El análisis de los datos indicó que los programas académicos estudiados tenían contenidos estrechamente relacionados con las necesidades de la profesión, es decir, una profesión con reconocimiento formal. En Canadá, según Verbeek (2011) sucede un fenómeno similar, aunque solo se han identificado dos maestrías relacionadas con GIRD, en 15 programas curriculares, entre certificados diplomados y licenciaturas (EMI, 2016). Es de destacar que en la difusión del Instituto de Manejo de Emergencias (EMI, por sus siglas en inglés) de los Estados Unidos, vinculado a la Agencia Federal de Manejo de Emergencias (FEMA, por sus siglas en inglés), solo se indican programas curriculares de "otros países", relacionados con Australia, Canadá, Nueva Zelandia, Turquía e Inglaterra. No se menciona a ningún país Latinoamericano. Las diferencias hablan por sí solas. Son concluyentes.

Con motivo del Día Internacional para la Reducción del Riesgo de Desastres el 13 de octubre de 2020, la Oficina de Naciones Unidas para la Reducción de Desastres (UNDRR, por sus siglas en inglés), publicó un muy interesante reporte que intituló "El costo humano de los desastres 20002019" (Mizutori y Debarati, 2020), que comparaba datos de registros de desastres y sus efectos de las décadas de 1990 a 1999 y de 2000 a 2019. Los registros de desastres crecieron en un 74\%, mientras que las muertes lo hicieron en un 33.6\%. La comparación, para esos mismos periodos, arrojó un incremento de $24 \%$ en personas afectadas y las pérdidas económicas en dólares de Estados Unidos, registró un incremento impactante de $82 \%$.

En una publicación reciente (Macías, 2020a, p.12) planteamos una interrogante que en sí misma engloba una compleja paradoja, que no puede responderse de manera sencilla: ¿por qué, a pesar del desarrollo científico-tecnológico en el conocimiento de las amenazas potencialmente desastrosas [avances en geociencias, en ingeniería de estructuras, conocimiento de fenómenos atmosféricos, etc.], de los esfuerzos internacionales por compartir esos avances y las inducciones de reorganización de las administraciones públicas, [DIRDN, Conferencias Internacionales de 
Acción para Reducir Desastres, de Kioto, Hyogo, Sendai], así como los esfuerzos en programas de inversiones para el "desarrollo" [el Banco Mundial tan solo en 2019 erogó casi 60 mil millones de dólares EEUU (Banco Mundial, 2019)], el número de desastres y sus pérdidas se han incrementado? Esta contribución trata de continuar ofreciendo elementos para buscar entender tal planteamiento, que es evidentemente paradojal, para el caso de los países latinoamericanos.

\begin{tabular}{lllll} 
Periodo & $\begin{array}{l}\text { Desastres } \\
\text { reportados }\end{array}$ & Total de muertes & Total de afectados & $\begin{array}{l}\text { Pérdidas económicas } \\
\text { Dólares }\end{array}$ \\
\hline $1980-1999$ & 4,212 & 1.19 millones & 3.25 billones & 1.63 trillones \\
\hline $2000-2019$ & 7,348 & 1.23 millones & 4.03 billones & 2.97 trillones \\
\hline
\end{tabular}

Comparativo de Impacto de Desastres 1980-1999 Vs. 2000-2019

Fuente: Mizutori y Debarati, (2020)

Fuente: Mizutori y Debarati, (2020)

" Para los propósitos de este reporte, el término 'desastre’ se reserva solo para los desastres relacionados con las amenazas

naturales, excluyendo a los desastres tecnológicos y biológicos.

** Los desastres relacionados con el clima, incluyen a los desastres categorizados como meteorológicos, climatológicos, o

hidrológicos.

*** Todas las cifras están ajustadas a la inflación en dólares de EEUU.

\section{REFERENCIAS}

AIC. (2010). Fundación de la asociación Las Damas de la Caridad. AIC-USA. https://aic.ladiesofcharity. us/files/2010/o2/Spanish-History-Book.pdf

Albala-Bertrand, J.M. (1993). Natural Disaster Situations and Growth: A Macroeconomic Model for Sudden Disaster Impacts. World Development, 21(9), 1417-1435.

Albala-Bertrand, J.M. (2014). Disasters and the Networked Economy. A Book Summary. Working Paper No. 718. Queen Mary University of London. https://www.researchgate.net/ publication/271441339_Disasters_and_the_Networked_Economy_A_Book_Summary

Amorim, H. (2015) El fin de las clases sociales. Estudios Latinoamericanos, 35, 15-37. http://dx.doi. org/10.22201/cela.24484946e.2015.35.46950

Banco Mundial. (2020). História. Banco Mundial. https://www.bancomundial.org/es/about/history

Banco Mundial. (2019, 11 de julio). Comunicado de Prensa. https://www.bancomundial.org/es/news/ press-release/2019/07/11/world-bank-group-financing-development-challenges-6o-billion-fiscalyear-2019.print

Bates, F., Dynes, R.R., \& Quarantelli, E.L. (1991). The Importance of Social Sciences to the International Decade for Natural Disaster Reduction. Disasters. The Journal of Disaster Studies and Management, 15(3), 288-289.

Borda, S. (2013). Estados Unidos o el último Estado hegemónico. Nueva Sociedad, 246. https://nuso. org/articulo/estados-unidos-o-el-ultimo-estado-hegemonico-el-poder-en-la-era-del-ascenso-y-laconsolidacion-del-resto-del-mundo/

Cavani, L. \& Revet, S. (2015). La cause des catastrophes. Concurrences scientifiques et actions politiques dans un monde transnational. Politix, 28(111/2015), 47-67. https://journals.openedition.org/ conflits/17693

CGCED. (2002). Natural Hazard Risk Management in the Caribbean: revisiting the challenge. Private Sector \& Infrastructure Department. Latin America and the Caribbean Region. The World Bank. http://www.oas.org/cdmp/riskmatrix/RM_CGCED.DOC

Christensen, C. (2019). Popper on Marx on History. Philosophy Now, 131. https://philosophynow.org/ issues/131/Popper_on_Marx_on_History

Dussel, E. (1966). Hipótesis para el estudio de Latinoamérica en la historia universal. CLACSO. http:// biblioteca.clacso.edu.ar/clacso/otros/20120408102154/latino.pdf

Dynes, R.R. (1994). Community Emergency Planning: False Assumptions and Innapropiate Analogies. International Journal of Mass Emergencies and Disasters, 12(1), 5-24.

EIRD. (2009). Oferta académica de Instituciones de Educación Superior (IES), relacionada directamente con la temática de Reducción del Riesgo de Desastres (RRD), en los países de América Latina en lengua española. Estrategia Internacional para la Reducción de Desastres REDULAC. Unidad Regional para las Américas. http://www.redulac.net/oferta_academica/images_oferta_academica/ Informe_oferta-academica.pdf 
EMI. (2016). Emergency and Disaster Management Programs in Other Countries. Emergency Management Institute. https://training.fema.gov/hiedu/collegelist/othercountries/

EOM. (2019). Las dictaduras en América Latina desde 1945. El Orden Mundial. https://elordenmundial. com/mapas/dictaduras-en-america-latina/

Freeman, P.K., Martin, L.A., Linnerooth-Bayer, J. Warner, K. \& Pflug, G. (2002). Sistemas Nacionales para la Gestión Integral del Riesgo de Desastres. Estrategias Financieras para la Reconstrucción en Caso de Desastres Naturales. Banco Interamericano de Desarrollo BID.

Garzón Real, B. (2016). Operación Cóndor. 40 años después. Centro Internacional para la Promoción de los Derechos Humanos (CIPDH). https://www.cipdh.gob.ar/promocion/ libro-operacion-condor-40-anos-despues/

GFDRR. (2014). Natural Disaster Risk Management. GFDRR https://www.preventionweb.net/ files/39526_ndrmpprogrambrochure.pdf

Gram-Skjoldager, K. (2019). Eric Drummond and his Legacies. The League of Nations and the Beginnings of Global Governance, written by David Macfadyen, Michael Davies, Marilyn Carr and John Burley. Diplomatica, 1(2), 308-310. https://brill.com/view/journals/dipl/1/2/article-p308_308. $\mathrm{xml}$ ?language $=\mathrm{en}$

Hallegatte, S., Vogt-Schilb, A., Bangalore, M. \& Rozenberg, J. (2017). Unbreakable: Building the Resilience of the Poor in the Face of Natural Disasters. International Bank for Reconstruction and Development \& The World Bank. https://documents.worldbank. org/en/publication/documents-reports/documentdetail/512241480487839624/ unbreakable-building-the-resilience-of-the-poor-in-the-face-of-natural-disasters

Henderson, L.J. (2004). Emergency and Disaster: Pervasive Risk and Public Bureaucracy in Developing Nations. Public Organization Review, 4, 103-119 https://doi.org/10.1023/B:PORJ.ooooo31624.46153.b2

Huffman, J.L. (1983). Government Liability and Natural Hazard Mitigation in Japan, The Soviet Union, China, New Zeland and the United States. International Journal of Mass Emergencies and Disasters, 1(3), 379-398.

IMF. (2016). El FMI y el Banco Mundial. Ficha Técnica. IMF. https://www.imf.org/es/About/Factsheets/ Sheets/2016/07/27/15/31/IMF-World-Bank

Jassir, M. (2015). Poder blando y diplomacia cultural: Elementos claves de políticas exteriores en transformaciones. Editorial Universidad del Rosario.

Kagarlitsky, B. (2015). Marxism in the Post-Globalization Era. Valdai Papers, 13. http://eng.globalaffairs. ru/number/Marxism-in-the-Post-Globalization-Era-19444

Kapucu, N. (2011). Developing Competency-Based Emergency Management Degree Programs in Public Affairs and Administration. Journal of Public Affairs Education. 17(4), 501-521.

Lavell, A. (2003). La gestión local del riesgo. Nociones y precisiones en torno al concepto y la práctica. Programa Regional para la Gestión del Riesgo en América Central. CEPREDENAC - PNUD. http:// www.disaster-info.net/lideres/portugues/brasil\%20o6/Material\%2oprevio/Allangestriesg.pdf

Lara, A., Concha M., F., González, S. \& Bucci A., F. (2014). Generación de una base de datos sobre la Oferta Académica en las áreas de Gestión del Riesgo de Desastres en América Latina y el Caribe. UNESCO. http://docplayer.es/9824111-Consultoria-generacion-de-una-base-de-datos-sobre-laoferta-academica-en-las-areas-de-gestion-del-riesgo-de-desastres-en-america-latina-y-el-caribe. html

Lara San Martín, A. (2016). Educación superior en América Latina y el Caribe para la Gestión y Reducción del Riesgo de Desastres: ¿Estamos preparando a nuestra población? Revista de Estudios y Experiencias en Educación, 15(29), 49- 63. http://www.rexe.cl/ojournal/index.php/rexe/article/ viewFile/318/319

Macías Chávez, K.C. (2015). El Neocolonialismo en Nuestros Días: La Perspectiva de Leopoldo Zea. Universitas Philosophica,32(65), 81-106. https://www.redalyc.org/pdf/4095/409543046004.pdf

Macías, J.M. (2016). Los modelos gubernamentales para enfrentar los problemas del riesgo-desastre (Defensa Civil, Protección Civil, Manejo de Emergencias). México. CIESAS-México. http://data. eap.cdmx.gob.mx/MaestriaGIRD/images/MISesin13_ModelosGubernamentalesLectura-JMMM_ Edicion2016.pdf

Macías, J.M. (2020a). El incremento de desastres y el rol del concepto de resiliencia. En Azamar, A. (Ed), Resistencias sociales y alternativas de sustentabilidad. Un análisis multidimensional (pp.187-212).

UAM-X. https://www.researchgate.net/publication/345777969 
Macías, J.M. (2020b). La ambigüedad de la Gestión Integral del Riesgo de Desastres. En López, L. \& Toscana, A. (Eds), El desastre y sus fronteras. Perspectivas locales (pp.19-44). UAM-X

Marks, C.A. (2005). Professional Competencies for the Master's Level Emergency Manager. Knowledge Systems Necessary for the Emergency Manager of the 21st Century. Federal Emergency Management Agency FEMA. https://training.fema.gov/hiedu/.../craigmarksprofessionalemgraddoc.doc

McClory, J. (2017). Soft Power y Latinoamérica. Foreign Affairs Latinoamérica. https://revistafal.com/ soft-power-y-latinoamerica/

Martínez-Méndez, F. \& López-Carreño, R. (2011). El sinsentido de hablar de literatura gris en la época 2.o. El Profesional de la Informacion, 20(6), 621-626. https://doi.org/10.3145/epi.2011.nov.03

Menéndez, E.L. (2018). Colonialismo, neocolonialismo y racismo. El papel de la ideología y de la ciencia en las estrategias de control y dominación. UNAM. http://www.nacionmulticultural.unam.mx/ portal/pdf/publicaciones_novedades_editoriales/libro_colonialismo_neocolonialismo_racismo.pdf

Mizutori, M. \& Guha-Sapir, D. (2020). The human cost of disasters: an overview of the last 20 years (2000-2019). CRED-EM-DAT \& UNDRR. https://www.undrr.org/publication/ human-cost-disasters-overview-last-20-years-2000-2019

Morales, D. (2017). El fin del ciclo hegemónico de Estados Unidos. Foreign Affairs Latinoamérica. https://revistafal.com/el-fin-del-ciclo-hegemonico-de-estados-unidos/

Narváez, L., Lavell, A. \& Pérez Ortega, G. (2009). La gestión del riesgo de desastres: un enfoque basado en procesos. PREDECAN. http://www.comunidadandina.org/predecan/doc/libros/ PROCESOS_ok.pdf

Newman, A. (2013). World Bank Insider Blows Whistle on Corruption, Federal

Reserve. The New American. https://thenewamerican.com/ world-bank-insider-blows-whistle-on-corruption-federal-reserve/

Nourigholamizadeh, E. (2020). Una Investigación Histórica sobre la Formación del Bloque Hegemónico de los Estados Unidos en las Américas. Revista Sociología Histórica, 10, 247-277. https://revistas. um.es/sh/article/view/402161

Ornelas, R. (2003). América Latina: territorio de construcción de la hegemonía. Revista Venezolana de Economía y Ciencias Sociales, 9(2), 117-135. https://www.researchgate.net/ publication/237026235_America_Latina_territorio_de_construccion_de_la_hegemonia

Parenti, S. (2011). Neocolonialism construction and solutions. University of Central Florida. https://stars.library.ucf.edu/cgi/viewcontent.cgi?referer=https://www.google. com/\&httpsredir=1\&article=2173\&context=honorstheses1990-2015

Tim, P. \& Roth, F. (2015). Global Disaster Politics Post Sendai. CSS Analyses in Security Policy, 173. https://www.preventionweb.net/files/44622_cssanalyse173en.pdf

Quijano, A. (1992). Colonialidad y Modernidad/Racionalidad. Perú Indígena, 13(29), 11-20. https://www. lavaca.org/wp-content/uploads/2016/o4/quijano.pdf

Quijano, A. (2014). Colonialidad del poder, eurocentrismo y América Latina. En Quijano, A. (Ed), Cuestiones y horizontes: De la dependencia histórico-estructural a la colonialidad/descolonialidad del Poder (pp.777-832). CLACSO. http://biblioteca.clacso.edu.ar/clacso/se/20140507042402/eje3-8.pdf

Rajamoorthy, T. (2004). Bretton Woods y el triunfo de la hegemonía de Estados Unidos. Third World Resurgence. Revista del Sur, 155-156. https://solidaridad.net/ bretton-woods-y-el-triunfo-de-la-hegemonia-de-estados-unidos-2384/

Dasgupta, R. (2018, 5 de abril). The demise of the nation state: After decades of globalization, our political system has become obsolete - and spasms of resurgent nationalism are a sign of its irreversible decline. The Guardian (UK). https://www.theguardian.com/news/2018/apr/o5/ demise-of-the-nation-state-rana-dasgupta

Rapoport, M. (1997). La globalización económica: Ideologías, realidad, historia. Ciclos, 7(2), 3-42. http://bibliotecadigital-old.econ.uba.ar/download/ciclos/ciclos_v7_n12_o1.pdf

Revet, S. (2011a). El mundo internacional de las catástrofes naturales. Política y Sociedad, 48(3), 537-554. http://dx.doi.org/10.5209/rev_POSO.2011.v48.n3.36424

Revet, S. (2011b). Vivre dans un monde plus sûr. Cultures $\mathcal{E}$ Conflits, 75(automne 2009), 33-51. https:// doi.org/10.400o/conflits.17693 
Ríos, J.L. (2018). Tres etapas del golpe "blando” en Brasil. Hacia una rearticulación social del capital. Revista de Ciencias Sociales, DS-FCS, 31,(43), 183-204. http://www.scielo.edu.uy/pdf/rcs/v31n43/16884981-rcs-31-43-183.pdf

Romano, S.M. (2017). La "Nueva Alianza” de Obama para América Latina: poder blando y poder duro en acción. Universidad de La Habana, 283, 59-78. http://scielo.sld.cu/pdf/uh/n283/uho5283.pdf

Sánchez-Mejorada, M.C. (2001). El Distrito Federal frente a la Segunda Guerra Mundial medidas e implicaciones. Relaciones, Estudios de historia y sociedad, $22(86)$.

Schemper, L. (2019). Science Diplomacy and the Making of the United Nations International Decade for Natural Disaster Reduction, Diplomatica, 1(2), 243-267. https://doi.org/10.1163/25891774-00102006

Sharp, G. (2011). De la dictadura a la democracia. Un Sistema Conceptual para la Liberación. The Albert Einstein Institution. https://www.sedh.gob.hn/documentos-recientes/59de-la-dictadura-a-la-democracia-un-sistema-conceptual-para-la-liberaci\% ${ }_{3} \% \mathrm{~B}_{3} \mathrm{n} /$ file

Stiglitz, J. (2019). El fin del neoliberalismo y el renacimiento de la historia. Nueva Sociedad. https://nuso. org/articulo/crisis-neoliberalismo-historia-elites-capitalismo-protestas/imprimir/

Victoriano Serrano, F. (2010). Estado, golpes de estado y militarización en américa latina: una reflexión histórico política. Argumentos (México), 23(64), 175-193. http://www.scielo.org.mx/pdf/argu/v23n64/ v23n64a8.pdf

Tanzi, V. (1998). The Demise of the Nation State? IMF Working paper. https://www.imf.org/external/ pubs/ft/wp/wp98120.pdf

Toussaint, E. (2007). Banco Mundial, el golpe de Estado permanente. La agenda oculta del Consenso de Washington. El Viejo Topo. http://www.cadtm.org/IMG/pdf/banco_mundial_abya_yala_ecuador.pdf

Toussaint, E. (2020). Domination of the United States on the World Bank. Series: 1944-2020, 76 years of interference from the World Bank and the IMF. CADTM. https://www.cadtm.org/spip. php?page=imprimer\&id_article=2194

Victoriano, F. (2010). Estado. Golpes de Estado y militarización en América Latina. Argumentos (México), 23 (64), 175-193. http://ref.scielo.org/j6zfcc

Van Niekerk, D., Nemakonde, L., Kruger, L. \& Genade, K. (2017). Community-based Disaster Risk Management. In Handbook of Disaster Research. Springer. https://www.researchgate.net/ publication/317411922_Community-based_Disaster_Risk_Management

Verbeek, M. (2011). The Evolving Roles of Emergency Managers. Canadian Risk and Hazards. (CRHNET). http://www.crhnet.ca/sites/default/files/library/Verbeek.pdf

Vitali, S., Glattfelder, J.B. \& Battiston, S. (2011). The Network of Global Corporate

Control. PLoS one, 6(10), 1-19. http://doi.org/10.1371/journal.pone.0025995

Wharton. (2000). World Bank/Wharton Conference on Innovations in Managing Catastrophic Risks: How Can They Help the Poor? Risk Management Review.

WHO/OMS. (2004). Definitions, Acronyms and Abbreviations related to inter-agency mechanisms in the field of emergency and humanitarian action. WHO/OMS. https://apps.who.int/disasters/ repo/13849_files/j/definitions_acronyms.pdf

Zabalo, P. (2002). Banco Mundial. Diccionario de Acción Humanitaria y Cooperación para el Desarrollo. Universidad del País Vasco. http://www.dicc.hegoa.ehu.es/listar/mostrar/21

Zapata, R. (2011). Experiencias exitosas en la transferencia del riesgo en América Latina y el Caribe. EIRD. 